\title{
Perfil e capacidade funcional em sujeitos com Doença de Huntington
}

\section{Profile and functional capacity in Huntington's disease subjects}

\author{
Hudson Azevedo Pinheiro ${ }^{1}, 2$ (D) Ana Maria Marques da Fonseca ${ }^{1}\left(\mathbb{D}\right.$, Elisângela Roseane da Silva Almeida ${ }^{1}(\mathbb{D}$, Reiny Kelly Costa \\ Rodrigues $^{1}$ (D) , Renan Fangel ${ }^{1}$ (D) , Flávia Perassa de Faria ${ }^{1}$ (D) \\ 1. Curso de Fisioterapia, Centro Universitário EuroAmericano de Brasília (UniEuro), Brasília-DF, Brasil. 2. Ambulatório de Referência em Geriatria e
} Gerontologia da Policlínica de Taguatinga/SRSSO/SESDF, Brasília-DF, Brasil.

\section{Resumo}

Objetivo: traçar o perfil e a capacidade funcional de sujeitos com Doença de Huntington (DH) atendidos em um ambulatório de fisioterapia do Distrito Federal. Método: trata-se de um estudo transversal em que foram aplicados um questionário para coletar informações gerais para traçar o perfil, além do índice de Barthel, escala Lawton para medir grau de independência para as atividades de vida diária, força de preensão palmar por meio do dinamômetro JAMARß, equilíbrio por meio da escala de equilíbrio de Berg, declínio cognitivo por meio da Montreal Cognitive Assessment (MoCA), e risco de disfagia utilizando a Eating assessment tool (EAT-10). Resultados: foram avaliados sete sujeitos, 58\% homens e 42\% mulheres com média de idade de 45,8 $\pm 10,5$ anos e tempo médio de diagnóstico de 7,5 $\pm 4,2$ anos. Todos apresentam redução da força de preensão palmar $(21,1 \pm 7,3 \mathrm{Kg} / \mathrm{F})$ e declínio cognitivo $(13,1 \pm 4,2$ pontos) no MoCA. Sobre o risco de cair, este se fez presente em todos os participantes com o risco de disfagia. Conclusão: os indivíduos com Doença de Huntington apresentaram déficit cognitivo, diminuição da força de preensão palmar e alterações de marcha; porém, ainda mantêm independência para as atividades básicas de vida diária, além de risco para a disfagia.

Palavras-chave: Doença de Huntington. Incapacidade Funcional. Perfil de Saúde.

\begin{abstract}
Objective: to outline the profile and functional capacity of subjects with Huntington's disease (HD) attended at a physiotherapy clinic in the Federal District. Methods: This is a cross-sectional study in which a questionnaire was used to collect general information to profile, besides Barthel index, Lawton scale to measure the degree of independence for the activities of daily life, palmar grip strength using the JAMAR® dynamometer, balance by means of Berg balance scale, cognitive decline by means of the Montreal Cognitive Assessment (MoCA), and risk of dysphagia using the Eating assessment tool (EAT-10). Results: Seven subjects were evaluated, $58 \%$ male and $42 \%$ female with mean age of $45.8 \pm 10.5$ years and mean diagnosis time of $7.5 \pm 4.2$ years. All presented reduced palmar grip strength $(21.1 \pm 7.3 \mathrm{Kg} / \mathrm{F})$ and cognitive decline $(13.1 \pm 4.2$ points) in MoCA. About the risk of falling this was present in all partcipants as well as the risk of dysphagia. Conclusion: The individuals with Huntington's disease presented cognitive deficit, decreased palmar grip strength and gait alterations, however still maintains independence for the basic activities of daily life, besides risk for dysphagia.
\end{abstract}

Keywords: Huntington's Disease. Functional Incapacity. Health Profile.

\section{INTRODUÇÃO}

A Doença de Huntington (DH), descrita pela primeira vez em 1872 por George Huntington, é uma doença neurodegenerativa, de herança autossômica dominante (mutações dinâmicas e repetidas de CAG) caracterizada, clinicamente, pela tríade de distúrbios de movimentos, problemas psiquiátricos (alteração de personalidade, depressão) e declínio cognitivo, podendo culminar com síndrome demencial ${ }^{1}$.

Com a evolução da doença, ocorre perda progressiva de neurônios, principalmente no núcleo caudado e no putâmem, que são responsáveis pela produção do neurotransmissor GABA; ocasiona o aparecimento de movimentos involuntários irregulares, coreia e distonia, sobrevindo de forma progressiva e degeneração mental ${ }^{2-4}$.
Geralmente, as pessoas com DH manifestam os sintomas entre 40 e 50 anos de idade, com tempo médio de sobrevida variando entre 15 e 20 anos, após o aparecimento dos primeiros sintomas, sendo a disfagia a principal complicação que pode causar perda ponderal, broncoaspiração e risco de morte ${ }^{5}$. A prevalência da doença varia conforme a região global, sendo escassos dados referentes à América Latina, e 13,7/100.000 habitantes no Canadá e 0,65/100.000 no Japão6 .

Levando em consideração as alterações geradas na funcionalidade desses sujeitos, a literatura aponta a necessidade da intervenção fisioterapêutica, objetivando, nesses indivíduos, as suas habilidades para a realização das atividades de vida diária (AVD), a adaptação de equipamentos disponíveis com 
foco na melhoria da capacidade funcional, minimizando, assim, risco de quedas ${ }^{7-8}$.

Com base no presente exposto, o objetivo deste estudo foi traçar o perfil e a capacidade funcional de indivíduos com $\mathrm{DH}$, atendidos em um ambulatório de fisioterapia do Distrito Federal, Brasil.

\section{MÉTODOS}

Foi realizado um estudo descritivo, série de casos, com amostra de conveniência oriunda do Projeto de Extensão "Ambulatório de Fisioterapia na Doença de Huntington" na Clínica Escola do Centro Universitário EuroAmericano de Brasília (UNIEURO), no Campus Águas Claras, em parceria com a Associação Brasil Huntington $(A B H)$, responsável pelo encaminhamento dos pacientes, entre os meses de março e junho de 2018.

Os critérios de inclusão foram sujeitos adultos e com $\mathrm{DH}$, residentes no Distrito federal, de ambos os sexos, cadastrados no site da $\mathrm{ABH}$ e que compareceram à entrevista agendada para iniciar acompanhamento fisioterapêutico.

Como critério de exclusão, deu-se a recusa em responder aos questionários aplicados ou o não comparecimento às consultas de admissão.

Este projeto foi Aprovado pelo Comitê de Ética em Pesquisa da UNIEURO, número do Protocolo 2.371.614, e todos os acompanhantes e/ou representantes legais preencheram e assinaram o Termo de Consentimento Livre e Esclarecido (TCLE). Inicialmente, foi aplicada uma ficha com a finalidade de coletar informações como sexo, idade atual, tempo de doença, histórico clínico, número de filhos, além de informações socioeconômicas para traçar o perfil desses sujeitos.

Para a avaliação de capacidade funcional, adotaram-se como instrumentos os testes a seguir.

Para a avaliação do comprometimento cognitivo, foi utilizado o Montreal Congnitive Assesment (MoCA), que é um instrumento de triagem breve para mensurar funções executivas como habilidades visoespaciais, nomeação, evocação, raciocínio abstrato, orientação no tempo e espaço, em que escores inferiores a 20 pontos são sugestivos de transtorno cognitivo $^{9-10}$

Para avaliar o grau de funcionalidade, utilizou-se a versão brasileira do índice de Barthel, instrumento que avalia o nível de independência na realização de dez atividades básicas da vida diária (ABVD), como comer, higienizar-se, usar sanitário, tomar banho, vestir-se, despir-se, controlar o esfincteriano, deambular, transferir-se da cadeira para a cama, subir e descer escadas, e escores inferiores a 60 pontos indicam dependência funcional ${ }^{11}$.

Para atividades instrumentais de vida diária (AIVD), foi utilizada a
Escala de Lawton que possui oito atividades em que o sujeito foi avaliado de acordo com o seu desempenho e/ou participação, necessidade de assistência, qualidade da execução e a iniciativa do sujeito ${ }^{12}$.

A escala de equilíbrio de Berg (EEB) foi utilizada para avaliar o equilíbrio dinâmico e estático dos indivíduos nas atividades do cotidiano, como ficar de pé, sentar-se, girar, transferir-se, alcançar, por meio de 14 tarefas distintas, sendo que 45 pontos já indicam predisposição para queda ${ }^{13}$.

Para mensurar a muscular, optou-se pela força de preensão palmar (FPP), uma vez que esse é um indicador da força muscular global, e foi utilizado o dinamômetro hidráulico Jamar ${ }^{\circledR}$, sendo adotado o protocolo recomendado pela American Society of Hand Therapists (ASHT) para a aquisição das informações ${ }^{14}$.

Para mensurar queixas relacionadas à disfagia, utilizou-se a Eating Assessment Tool (EAT-10), um questionário de 10 questões autoaplicáveis, que deverão ser respondidas de modo subjetivo pelo paciente, cujas respostas variam em uma escala de 0 a 4 , em que escores superiores a quatro pontos indicam disfunção $0^{15}$.

Para a avaliação da marcha, foi utilizada a escala de Classificação Funcional da Marcha Modificada (CFMM), em que o paciente é classificado em uma escala de zero a cinco pontos, sendo que a pontuação zero significa que não realiza marcha; pontuação um, marcha terapêutica não funcional; pontuação dois, marcha domiciliar; pontuação três, deambula pela vizinhança; pontuação quatro, marcha comunitária e pontuação cinco, marcha normal ${ }^{16}$.

Para análise dos dados, utilizou-se o software Statistical Package for the Social Sciences (SPSS) versão 22, em que as variáveis quantitativas foram descritas por meio de mediana e valores mínimos e máximos, e as variáveis categóricas por frequências absoluta e relativa.

\section{RESULTADOS}

Após contato com a ABH para divulgação do presente projeto, foram captados sete sujeitos oriundos de quatro famílias distintas cujas características estão disponíveis na tabela 1.

Observou-se que cinco sujeitos são casados $(71,5 \%)$ e que todos os indivíduos têm filhos, podendo contribuir com risco de novos casos de DH nas próximas gerações.

A respeito da capacidade funcional, os dados são apresentados na tabela 2; observa-se que todos ainda são independentes para as ABVD com escores superiores a 60 pontos no índice de Barthel, apesar de apresentarem redução da força de preensão palmar além de declínio cognitivo, apontado pelo MoCA, além das queixas relacionadas à disfagia mensuradas por meio da escala EAT-10. 
O risco de cair apenas um sujeito apresentou escores superiores a 45 pontos na $E E B$, e este é justamente o que ainda apresenta marcha comunitária.

Tabela 1. Aspectos socioeconômicos dos sujeitos com doença de Huntington atendidos em um ambulatório de Fisioterapia do Distrito Federal, 2018.

\begin{tabular}{lrrr}
\hline Variável & N & \% & Média \pm DP \\
\hline Idade (anos) & & & $45,8 \pm 10,5$ \\
\hline Tempo de diagnóstico (anos) & & & $7,5 \pm 4,2$ \\
Tem filhos? & & & $2,5 \pm 1,5$ \\
Sexo & 03 & & \\
$\quad$ Feminino & 04 & \\
Masculino & & \\
Estado Civil & & & \\
Solteiro & 03 & \\
Casado & 05 & 71,5
\end{tabular}

Escolaridade

$\begin{array}{lll}\text { Ensino Médio } & 04 & 57,2 \\ \text { Ensino Superior } & 03 & 42,8\end{array}$

\begin{tabular}{lll} 
Renda $^{£}$ & & \\
Até 2 salários & 01 & 14,3 \\
Entre 3 e 5 salários & 04 & 57,2 \\
Mais de 6 salários & 02 & 28,5 \\
\hline
\end{tabular}

f=salário-mínimo em vigor em 2018 é de $\mathrm{R} \$ 937,00$.

Tabela 2. Capacidade funcional em sujeitos com Doença de Huntington atendidos em um ambulatório de fisioterapia.

\begin{tabular}{lrrr}
\hline Variável & Mediana & $\begin{array}{r}\text { Valores } \\
\text { Mínimos }\end{array}$ & $\begin{array}{r}\text { Valores } \\
\text { Máximos }\end{array}$ \\
\hline Índice de Barthel & 83 & 75 & 97 \\
Escala Lawton & 16 & 13 & 19 \\
Força de Preensão Palmar & 21 & 12 & 23 \\
MoCA & 13 & 9 & 17 \\
EEB & 41 & 35 & 45 \\
EAT-10 & 7 & 5 & 9 \\
\hline
\end{tabular}

MoCA = Montreal Cognitive Assesment; $\mathrm{EEB}=$ Escala de equilíbrio de Berg; EAT-10= Eating Assesment Tool; $\mathrm{CIF}=$ Classificação Internacional de Funcionalidade.

\section{DISCUSSÃO}

Observou-se, pelos dados colhidos, que todos os indivíduos têm filhos, o que remeteu ao questionamento sobre o aconselhamento genético, cujo objetivo é fornecer informações detalhadas sobre determinada condição às famílias, com explicação dos riscos genéticos, além da importância de os parentes em risco submeterem-se a exames genéticos relacionados ao problema, caso o desejarem, uma vez que a hereditariedade é fator preponderante na determinação do fator de risco para $\mathrm{DH}$, com probabilidades altíssimas para a descendência ${ }^{17}$.

De acordo com o relato dos participantes, antes de apresentarem os primeiros sintomas da $\mathrm{DH}$, possuíam vínculo empregatício ou desenvolviam uma função remunerada na sociedade até o surgimento dos primeiros sintomas, como falta de destreza e precisão de movimentos distais, presença de discinesia (coreia e distonia) alterações sutis na marcha, além do declínio cognitivo, o que corrobora o afastamento do trabalho e, consequentemente, de uma vida social produtiva ${ }^{18}$.

Quanto ao risco de quedas, Hausdorff et al.(2007) relataram que a coreia afeta o centro de gravidade dos pacientes com $\mathrm{DH}$ durante a marcha e que os movimentos involuntários, se muito intensos, podem prejudicar o equilíbrio, resultando na queda do paciente por não conseguir o necessário ajuste postural antecipatório e compensatório que evitaria a queda ${ }^{19}$.

No tocante à idade, encontra-se uma média de 41,5 anos, com diferença média de idade de 17,5 anos, circunscrevendo os indivíduos em patamar de meia idade, notadamente, a faixa etária de maior incidência da $\mathrm{DH}$ em que, aproximadamente, $80 \%$ dos casos situam-se em pacientes de meia idade, uma vez que, aproximadamente $10 \%$ correspondem à forma juvenil e igualmente, $10 \%$ correspondem à forma sexagenária ${ }^{20}$.

Não obstante, o fato de haver uma diferença média de 7,5 anos de diagnóstico da $\mathrm{DH}$ e considerando o desvio-padrão de $\pm 4,2$ anos, com exceção do participante número sete (paciente acamado), os demais, apesar da diferença no tempo de diagnóstico e fase de evolução da doença, são independentes para as ABVD. Entretanto, os pacientes com DH apresentam prejuízo nessa área somente em estágios mais avançados, diferentemente do que ocorre com as AIVD que dependem diretamente da capacidade cognitiva ${ }^{21-22}$.

Outro fator importante a ser observado nos pacientes com DH é a disfagia, que é a principal causa de morte para esses pacientes, devido à perda da capacidade de deglutição, levando à morte por asfixia ou pneumonia aspirativa que ocorre nos estágios mais avançados da doença. Entretanto, os problemas de deglutição podem ser vistos em estágios inicias da $\mathrm{DH}$, mesmo que nenhum comprometimento motor seja evidente ${ }^{23}$.

Uma doença progressiva e que acarreta disfunções significativas que acometem a capacidade funcional além de risco aumentado de comorbidades e mortalidade, a DH, historicamente, traz um estigma para os portadores do gene e suas famílias que, muitas vezes, têm receio em buscar, precocemente, o aconselhamento genético por medo, e essa incerteza em saber se possuem ou não o gene, cria um fantasma que segrega e martiriza principalmente os familiares mais próximos, responsáveis pelo cuidado direto e a dúvida de ser o próximo acometido ${ }^{24}$. 
Sobre a força muscular, a amostra apresenta escores inferiores ao referencial de normalidade, o que pode levar à diminuição na mobilidade e estabilidade e justificar as dependências para as ABVD e risco de quedas ${ }^{25}$. No estudo de Cruickshank et al., foi proposto um protocolo multiprofissional cujo objetivo era melhorar mobilidade, força muscular e humor em 22 sujeitos com DH e, após 36 semanas de acompanhamento, observaram efeitos positivos que foram gerados por meio desse protocolo, que incluía o treino de força muscular como parte do tratamento ${ }^{26}$.

As disfunções cognitivas estão associadas aos avanços da DH, contextualizadas aqui com alterações de memória, e, principalmente, funções executivas (atenção, cálculo, inibição, percepção, abstração), que se associam a desordens psiquiátricas como apatia, depressão e, principalmente, compulsão ${ }^{27}$. No presente estudo, escores baixos do MoCA em que se verificam as alterações de funções executivas se associam com os achados da Lawton, indicando dependência nas atividades instrumentais de vida diária, prejudicando as capacidades laborativas, lazer e participação social.

A literatura carece de informações mais detalhadas sobre as disfunções geradas pela DH para a elaboração de estratégias fisioterapêuticas aplicáveis para o incremento de força muscular, minimizar risco de quedas, além de mecanismos para intervir nas desordens motoras como a coreia, fazendo-se necessários mais estudos sobre essa temática.

\section{CONCLUSÃO}

Os indivíduos com doença de Huntington apresentaram deficit cognitivo, diminuição da força de preensão palmar, presença de disfagia e alterações de marcha que os restringiram ao domicílio; porém, ainda mantêm independência para as atividades básicas de vida diária.

Agradecimento: A Associação Brasil Huntington ( $\mathrm{ABH}$ ) pela confiança e parceria, pela divulgação do presente projeto e encaminhamento de pacientes.

\section{REFERÊNCIAS}

1. Quagliato EMAB, Marques MGN. Doença de Huntington. São Paulo: Atibaia; 2009.

2. Joel D. Open interconnected model of basal ganglia-thalamocortical circuitry and its relevance to the clinical syndrome of Huntington's disease. Mov Disord Off J Mov Disord Soc. 2001; 16(3): 407-23.

3. Bano $D$, Zanetti $F$, Mende $Y$, Nicotera P. Neurodegenerative processes in Huntington's disease. Cell Death Dis. 2011; 2(11): e228.

4. Ross CA, Tabrizi SJ. Huntington's disease: from molecular pathogenesis to clinical treatment. Lancet Neurol. 2011; 10(1): 83-98.

5. Barsottini OGP. Doença de Huntington: o que é preciso saber. Einstein. 2007; 5(3): 83-8.

6. Baig SS, Strong M, Quarrell OWJ. The global prevalence of Huntington's disease: a systematic review and discussion. Neurodegenerative disease management. 2016; 6(4): 331-43

7. Bittencourt A, de Lima RLLF, de Azevedo Moreira LM. Percepções sobre a doença de Huntington e realização de testes preditivos em indivíduos com história da doença na família. Rev Ciências Médicas e Biológicas. 2010; 9(2): 126-9.

8. Walter J, Grave MTQ, Périco E. Avaliação das habilidades psicomotoras e da motricidade global em paciente portadora da Doença de Huntington. ComScientiae Saúde; 2009; 8(4):655-63.

9. Freitas S, Simões MR, Martins C, Vilar M, Santana I. Estudos de adaptação do Montreal Cognitive Assessment (MoCA) para a população portuguesa. Aval. psicol. Dez 2010; 9(3): 345-357.

10. Bezdicek O, Majerova V, Novak M, Nikolai T, Ruzicka E, Roth J. Validity of the Montreal Cognitive Assessment in the detection of cognitive dysfunction in Huntington's disease. Appl Neuropsychol Adult. Taylor \& Francis; 2013; 20(1): 33-40.

11. Minosso JSM, Amendola F, Alvarenga MRM, Campos Oliveira MA. Validação, no Brasil, do Índice de Barthel em idosos atendidos em ambulatórios. Acta paul enferm. 2010 Mar-Abr; 23(2):218-23.

12. Del Duca GF, Silva MC, Hallal PC. Incapacidade funcional para atividades básicas e instrumentais da vida diária em idosos. Rev Saude Publica. 2009 Out; 43(5):796-805. doi: 0.1590/\$0034-89102009005000057.

13. Miyamoto ST, Lombardi I Júnior, Berg KO, Ramos LR, Natour J. Brazilian version of the Berg balance scale. Braz J Med Biol Res. 2004 Sept; 37(9):141121. doi: http://dx.doi.org/10.1590/S0100-879X2004000900017.

14. Martin FG, Nebuloni CC, Najas MS. Correlação entre estado nutricional e força de preensão palmar em idosos. Rev bras geriatr e gerontol. $2012 \mathrm{Jul}-\mathrm{Sept}$; 15(3): 493-504. doi: http://dx.doi.org/10.1590/S1809-98232012000300010.

15. Gonçalves MIR, Remaili CB, Behlau M. Cross-cultural adaptation of the Brazilian version of the Eating Assessment Tool-EAT-10. Codas. 2013 Dez; 25(6): 601-604.

16. Viosca E, Martínez JL, Almagro PL, Gracia A, González C. Proposal and validation of a new functional ambulation classification scale for clinical use. Arch Phys Med Rehabil. 2005 Jun; 86(6): 1234-8.

17. Ximenes BA de A, Teixeira EH. Doença de Huntington: aspectos diagnósticos e implicações éticas. Rev ciênc méd. 2009; 18(5/6): 287-91.

18. Januário $F$, Júlio $F$, Januário $C$. Qualidade de vida na doença de Huntington: que sintomas a influenciam. Sociedade Portuguesa de Neurologia. Sinapse. 2011; 11(1): 12-16.

19. Hausdorff JM. Gait dynamics, fractals and falls: finding meaning in the stride-to-stride fluctuations of human walking. Hum Mov Sci. 2007 Aug; 26(4): 555-589.

20. Lee J-M, Ramos EM, Lee J-H, Gillis T, Mysore JS, Hayden MR, et al. CAG repeat expansion in Huntington disease determines age at onset in a fully dominant fashion. Neurology. 2012 Mar; 78(10): 690-695.

21. Hamilton JM, Salmon DP, Corey-Bloom J, Gamst A, Paulsen JS, Jerkins S, et al. Behavioural abnormalities contribute to functional decline in Huntington's disease. J Neurol Neurosurg Psychiatry. 2003 Jan; 74(1): 120-122.

22. Paulsen JS, Langbehn DR, Stout JC, Aylward E, Ross CA, Nance M, et al. Detection of Huntington's disease decades before diagnosis: the Predict-HD study. J Neurol Neurosurg Psychiatry. 2008 Aug; 79(8): 874-80.

23. Heemskerk A-W, Roos RAC. Dysphagia in Huntington's disease: a review. 


\section{Capacidade funcional na Doença de Huntington}

Dysphagia. 2011 Mar; 26(1):62-6. doi: 10.1007/s00455-010-9302-4.

24. Wexler A. Stigma, history, and Huntington's disease. Lancet. 2010 Jun; 376(9734): 18-9. doi: https://doi.org/10.1016/S0140-6736(10)60957-9.

25. Moreira D, Álvarez RR, de Gogoy JR, Cambraia ADN. Abordagem sobre preensão palmar utilizando o dinamômetro Jamar: uma revisão de literatura. R.Bras. Ci e Mov. Jun 2003;11(2): 95-100
26. Cruickshank TM, Reyes AP, Penailillo LE, Pulverenti T, Bartlett, D. M., Zaenker, et al. Effects of multidisciplinary therapy on physical function in Huntington's disease. Acta Neurol Scand. 2018 Dec;138(6): 500-7. doi: 10.1111/ane.13002.

27. Julayanont P, Heilman KM, McFarland NR. Early-motor phenotype relates to neuropsychiatric and cognitive disorders in huntington's disease. Mov Disord. 2020 Jan. doi: $10.1002 /$ mds. 27980

Como citar este artigo/How to cite this article:

Pinheiro HA, Fonseca AMM, Almeida ERS, Rodrigues RKC, Fangel R, Faria FP. Perfil e capacidade funcional em sujeitos com Doença de Huntington. J Health Biol Sci. 2020 J; 8(1):1-5. 